\title{
Molecular targets on antiepileptogenic therapy research: deconstructing the network for translational purposes
}

\begin{abstract}
The current pharmacological approaches on Epilepsy, the AEDs, 'antiepileptic drugs', are mostly seizure suppressing. However on the last decade, a greater attention has been given to research focusing the epileptogenesis process, the mechanism whereby a neuronal network develops recurrent spontaneous epileptic seizures. On this regard a new term, alternatively to the classical AEDs, has been proposed. The 'Anti-seizure drugs' are those compounds with an active intervention on the disease progression and that have shown a sustained modulatory effect over network hyperexcitability. Experimental studies on anti-seizure drugs development focus on cell processes as synaptic plasticity, inflammatory and immune responses and also glial intervention on neurotransmission, with the aim of the discovery of potential molecular targets playing a role on the etiology of epilepsy syndromes. Here, there are summarized the most prominent findings compiled from research studies on the last decade over in vivo and in vitro experimental models. However, these models are far for meeting the clinical needs on Epilepsy therapeutics. Future research approaches should have a strong translational component with the aim of identifying molecular targets, which, along with the understanding of regional interactions at the beginning and during the evolution of seizures, would help to unravel the epileptogenesis mechanisms and thus leading to the identification of more effective therapies.
\end{abstract}

Keywords: Antiepileptogenic drugs; Epilepsy therapeutics; Synaptic plasticity; Long term potentiation; Glutamate receptors; Immune/inflammatory response; Gliosis; Gene therapy; miRNAs targeting
Volume 2 Issue 2 - 2015

Freire-Cobo Carmen

Research Scientist, Georgia Regents University, USA

Correspondence: Freire-Cobo Carmen, Research Scientist, Brain and Behavior Discovery Institute, Georgia Regents University, Augusta, GA 30912, USA, Email cfreirecobo@gru.edu

Received: March 22, 2015 | Published: April 21, 2015
Abbreviations: AED, antiepileptic drugs; PSD, post-synaptic density; LTP, long term potentiation; AMPAR, $\alpha$-amino-3-hydroxy-5-methyl-4-isoxazole propionate receptor; NMDAR, n-methyl-d-aspartate receptor; $\mathrm{GABA}_{\mathrm{A}} \mathrm{R}, \gamma$-aminobutyric acid receptor A; PSD-95, post-synaptic density protein-95; ERK, extracellular-signal-regulated kinase; MAPK, mitogen-activated protein kinase; CREB, cAMP response element-binding protein; IL, interleukin; TNF, tumor necrosis factor; ATL, anterior temporal lobectomy; LTP, long term potentiation; TLR4, toll-like receptor-4; TBI, traumatic brain disorders; TLE, temporal lobe epilepsy; GFAP, glial fibrillary acidic protein

\section{Introduction}

Epilepsy is the second most common neurological disorder, affecting more than 50million people worldwide. ${ }^{1}$ Although new therapies in the epilepsy treatment have been introduced as a result of the preclinical research over the last two decades, seizures are not yet controlled in all patients. Current pharmacological approaches on Epilepsy are mostly seizure suppressing and the associated comorbidities suppose a serious withdrawal for patients, burdening their life quality. ${ }^{2}$ To address this, a greater attention has been given on research focusing epileptogenesis. This mechanism is defined as the process whereby a neuronal network develops recurrent spontaneous epileptic seizures and at the same time, the cause of seizures becoming more severe and frequent in chronic epilepsy. ${ }^{3}$ On this regard, it has been recently proposed an alternative terminology to define the current therapeutical approaches on Epilepsy and to be differenced from the classical designation of AED, 'antiepileptic drugs' on the symptomatic treatment. The term 'anti-seizure drugs', describe those compounds with an active intervention on the disease development and that have shown a sustained modulatory effect on the predisposition to generate spontaneous recurrent seizures in vivo and in vitro experimental models. ${ }^{4}$ Herein, there are summarized the main potential molecular targets, extracted from these research models and proposed to play a key role on the epileptogenic process. These studies have the ultimate goal of the development of disease biomarkers on future experimental designs that would allow for the identification of patients at risk of developing epilepsy or for monitoring disease progression and prognosis on treatment outcome.

\section{Discussion}

\section{The need of disassembling the network for the upgra- de on epilepsy therapeutics}

The classic traditional approach on epilepsy treatment has always focused on restoring the normal function on epileptic networks. Seizures occur in groups of neurons and involve complex interactions across several regions. Targeting recurrent hyperexcitability and thus stopping the number and intensity of episodes that utterly accelerate the development of disease, would also delay its direct consequences on appearance of disease comorbidities. ${ }^{3}$ Electrical brain stimulation, including the novel responsive stimulation (RNS), is used to control seizures and achieve modulation of disease symptoms, particularly on refractory and medication-resistant seizures, where no other option is available in the current therapeutics. ${ }^{5}$ The benefits of this approaches 
increase over time, but complications from this techniques are associate with the implantation procedure and hardware control, so further preclinical and clinical experience is needed for its completely safe use. In addition, Epilepsy surgery has the potential to cure seizures. The anterior temporal lobectomy (ATL), is the most common surgical approach where both, mesial and lateral temporal lobe structures are resected. However, it often comes associated with the impairing on cognitive function, especially verbal memory. ${ }^{6}$ Overall, the lack of precise information on the mechanisms of Epilepsy etiology and onset reveals a big gap on its treatment, even leading to hypothesize whether only targeting networks should be the main focus on Epilepsy therapeutics, taking also into account the invasive of the currently available resources with that aim. There is an urge for attributing equal attention to alterations at the molecular level in single neuronal populations on the time course of the disease progression. During the last decades, a number of experimental models have been developed on the study of the mechanism underlying the epileptogenesis process over time. Either by electrical or chemical induction of recurrent spontaneous seizures, chronic animal models of Epilepsy are the most important prerequisite to achieve the discovery and development of the antiepileptogenic and disease-modifying drugs. Previous reports also demonstrate that drug testing in those chronic models, in comparison with acute models of Epilepsy, shed results that are more predictive of clinical efficacy and adverse effects. ${ }^{7}$

\section{Targeting plasticity mechanism on synapsis}

Suggested by experimental evidence, the epileptogenic mechanism involves a cascade of molecular, cellular and neuronal network alterations following the trigger of an initial insult such as a traumatic brain injury and/or status epilepticus. These events, mostly alterations in plasticity of synapsis, ultimately causing dendritic tree rearrangement and network reorganization, take place in different temporal stages constituent of this pathophysiological mechanism and are consider factors of susceptibility on the onset of Epilepsy, classified on a temporal scale:

a. Acute early changes, including: rapid alterations to ion channel kinetics as a result of membrane depolarization, posttranslational modifications to existing functional proteins and immediate early genes, described to be transcriptionally activated (Fos, Jun, Egr1, Egr4, Homer1, Nurr77 and Arc).

b. Subacute changes, as those including neuronal death and activation of inflammatory cascades.

c. The chronic changes including anatomical changes within the network, such as: neurogenesis, mossy fiber sprouting, network reorganization and gliosis. ${ }^{8}$

A large number of those elements are identified as the molecular targets on the synaptic plasticity process induced on the latent period that follows an initial insult in epileptogenesis (Table 1). Such changes are similar to alterations taking place after the induction of long term potentiation (LTP) to increase synaptic efficiency. The mechanism involves key components of excitatory and inhibitory synapsis and includes among others: expansion of the PSD, enlargement of synapsis and clustering of excitatory neurotransmitter receptors. ${ }^{9}$ The main underlying molecular mechanism is thought to be prompted by calcium influx into the cell through ligand and voltage activated receptor channels. Its consequent action on phosphatases and kinases would in turn, alter ion channel and neurotransmitter receptor function and density on the neuron by altering their conductance and/or modulation of exo/endocytosis and membrane trafficking mechanism. On this regard, not only molecular targets like ion channels, ${ }^{10-12}$ Glutamate AMPA and NMDA and GABA receptors ${ }^{13-16}$ but also key proteins responsible for postsynaptic structure rearrangement after brain injury are been focus of study. Some examples include: cytoskeletal proteins, like F-actin; $;^{17,18}$ accessory proteins for receptor and vesicle trafficking, like Stargazing and Synaptin;9,17,19 Scaffold proteins like PSD-95, Kalirin-7/Trio among others. ${ }^{20,21}$ In addition, at the intracellular level, there are also been reported elements responsible for postsynaptic cell transduction pathways taking part on the epileptogenesis mechanism highlighting the ERK/MAP kinase cascade and the activation of CREB-based gene expression. ${ }^{7-9}$

Table I Molecular targets in epileptogenesis therapy- Summary of cell elements showing alterations experimental models of epileptogenesis and proposed as factors on seizure suscitability

\section{Neurotransmitter transporters, receptors and ion channels}

P-GluRI (Ser83I and Ser845);

P-GluR2 (Ser880)

Glutamate $^{8,9,13-15,38}$

$\downarrow$ AMPAR-GluR2; NMDAR-NR2B and NRI

(early-life seizures)

$\uparrow \mathrm{mGluRI}$

$\mathrm{GABA}_{\mathrm{A}} \mathrm{R}$ dephosphorylation

$\mathrm{GABA}^{8,9,16,38}$

$\uparrow \mathrm{GABA}_{\mathrm{A}} \mathrm{R} \propto \mathrm{I}$

$\downarrow \mathrm{GABA}_{\mathrm{A}} \mathrm{R} \alpha 4$ (immature brain)

Dopamine $e^{9,38-40}$

$\uparrow$ DIR; DAR2

Kv2.I dephosphorylation

$\mathrm{HCN}$ channels (increase/decrease in

Other Channels ${ }^{10,11,41,42}$ different animal models)

$\downarrow \mathrm{CBI}$

$\uparrow \mathrm{NKCCI}$

Glial Transporters ${ }^{7,25,26}$

$\downarrow$ EAATI; EAAT2

$\uparrow$ DAT

Immune/Inflamatory response

$\uparrow$ IL-I $\delta$; IL-I $\beta$; IL-2; IL-6;TNF- $\alpha$

Citokines \& Growth

Factors ${ }^{7,10,25,26}$

$\uparrow \mathrm{NFkBI}$; BDNF;TLR4

$\uparrow$ GFAP

$\uparrow$ Somatostatin; Neuropeptide Y; Galanin; Dynorphin;

Encephalin; Substance P; Cholecystokinin; VIP

AMPAR, $\alpha$-amino-3-hydroxy-5-methyl-4-isoxazole propionate receptor BDNF, brain-derived Neurotrophic factor; $\mathrm{CBI}$, cannabinoid type I receptor; COX-2, cyclooxygenase 2; DAT, dopamine transporter; EAAT, excitatory amino acid transporter; GABAAR, $\gamma$-aminobutyric acid receptor a; GFAP, glial fibrillary acidic protein; $\mathrm{HCN}$, hyperpolarization-activated cyclic nucleotidegated; IL, interleukin; Kv, voltage-gated potassium channel; NFKB, nuclear factor $\mathrm{Kb}$; NKCCI, sodium-potassium-chloride co-transporter I; NMDAR, n-methyl-d-aspartate receptor;TLR4, toll-like receptor-4;TNF, tumor necrosis Factor;VIP, vasoactive intestinal polypeptide. 
Targeting immunity/inflammation mechanisms and glial response

Experimental and clinical data suggest, ${ }^{22,23}$ that both native and adaptive immunity might contribute to the pathogenic processes associated with some seizures, particularly with refractory Epilepsies. Also, paradoxically, it has been reported that some routinely AEDs used to treat epilepsy, like Vigabatrin and Topiramate, can cause changes in the immune system by altering either cellular or humoral responses exacerbating this malfunction. ${ }^{22}$ The beneficial effect of the introduction, as part of some refractory epilepsy therapies, of compounds like corticosteroids, immunoglobulins and monoclonal antibodies (Rituximab, Efalizumab and Natalizumab) or immunosupressors (Cyclosporine A, Tacrolimus and Rapamycin), confirms the important influence on epileptogenesis of this associated processes and thus postulating new molecular targets on the initiated immune/inflammatory cascades. ${ }^{19,24,29}$

On the other hand, it is well known that glial cells play an important role in generating seizures by modulating synaptic transmission and, in the long term, contributing to the epileptic process by inducing inflammatory milieu around neurons. Particularly, astrocytes and microglia, are considered key players on initiating this inflammatory response after a brain insult. The increase in astrocyte reactivity in response to injury (astrogliosis) involves, according to experimental data: ${ }^{25-27}$ changes in morphology, increased expression of the intermediate filament proteins, glial fibrillary acidic protein (GFAP) and Vimentin and above all, the secretion of inflammatory mediators and growth factors such as: toll-like receptor-4 (TLR4); nuclear factor$\kappa \mathrm{B}(\mathrm{NF \kappa B})$; and cytokines (IL-1 $\beta$, TNF- $\alpha$ and IL-6) that ultimately, can influence the local microenvironment and thus determine the extent of damage and subsequent repair.

Also, related with glial involvement on Epilepsy, excitotoxicity is another common mechanism of secondary brain injury that has mainly being described in Traumatic Brain disorders (TBI), but as well suggested in epileptogenic experimental models. ${ }^{25,26}$ Astrocytes, through their glial transporters, are essential for glutamate re-uptake from synapses, preventing from excessive extracellular glutamate accumulation. Down-regulation in glial expression of glutamate EAAT1 and EAAT2 transporters has been observed in TBI lesional and peri-lesional regions. This accumulation of glutamate in extracellular areas, in addition to the previously described changes on expression and redistribution pattern of excitatory glutamate receptors to the extrasynaptic compartment on the cell membrane, would facilitate the synchronization of hyperexcitable networks as the main consequence of brain circuitry rearrangement after the epileptogenic insult. ${ }^{8,19}$ On the same line, Neuropeptides are signaling molecules participating in the modulation of synaptic transmission. These peptides are released after neural excitation in the extracellular space and exert their action on G-protein coupled receptors pre and postsynaptically. A number of neuropeptides have receptors involved in epilepsy (Table 1) and many of them are considered to participate in endogenous neuroprotective actions. Thus, neuropeptide modulators acting on receptors of hippocampal neuropeptides are ideal candidates to influence epileptic tissue overexcited during seizures receiving the largest attention as potential anti-epileptic targets. ${ }^{27}$

\section{Targeting 'epileptic' genes and altered microRNA in- duction}

The massively parallel gene sequencing studies during the last years, have shown results highlighting the importance of gene mutation in the etiology of Epilepsy. These data coupled to clinic genetic studies suggest that, in contrast to the traditional belief, any form of epilepsy is likely to have genetic basis. ${ }^{28}$ This leads to two important repercussions, firstly, a new paradigm on the clinical research for the potential detection of 'pro-epileptic' genes, providing with a genetic diagnosis and thus allowing for adequate counseling and prevention and thus important therapeutical implications for patients. ${ }^{29-33}$ And secondly, it opens the horizon for the introduction of gene therapy for Epilepsy treatment considering the fact that a reported $25 \%$ of patients do not respond satisfactorily to antiepileptic medication and only a minority with pharmacoresistant epilepsy is eligible for potential curative surgery. ${ }^{28}$ Several studies have already explored gene therapy as a treatment strategy and although several molecular genetic test are already in clinical use for diagnosis of epilepsy and their number is rapidly growing (Table 2), to move forward, these approaches must be tested in experimental models that best replicates the clinical situation in which invasive gene therapy is warranted, overall redirecting efforts to treating types of epilepsy that are more disruptive and refractory to known treatments.

Table 2 Targeted genes and miRNAs

\begin{tabular}{|c|c|c|}
\hline \multicolumn{3}{|c|}{ Genes and miRNAs } \\
\hline Target gene & \multicolumn{2}{|l|}{ Protein altered } \\
\hline SCNIA ${ }^{28,29}$ & \multicolumn{2}{|c|}{ Mutations in Sodium channel type $I \alpha$} \\
\hline SLC2A ${ }^{28,42}$ & \multicolumn{2}{|l|}{ Mutations in GLUTI } \\
\hline PCDHI $9^{28,30}$ & \multicolumn{2}{|c|}{ Mutations in Protocadherin 19} \\
\hline GRIN2A 28,32 & \multicolumn{2}{|c|}{ Mutations in NMDAR subunitSI } \\
\hline DEPDC $5^{28,31}$ & \multicolumn{2}{|c|}{ Mutations in DEP domain-containing 5} \\
\hline $\mathrm{KCNTI}^{28,33}$ & \multicolumn{2}{|c|}{$\begin{array}{l}\text { Mutations } n \text { potassium channel subfamily } T \\
\text { member I }\end{array}$} \\
\hline Targeted miRNA & Cell targets & Effect \\
\hline $\operatorname{miR}-34 a^{35}$ & $\begin{array}{l}\text { Bcl-2, CDK4, SIRTI, } \\
\text { Map3k9, Syt }\end{array}$ & $\uparrow p 53, p 73$ \\
\hline \multirow{2}{*}{$\operatorname{miR}-132^{34}$} & \multirow{2}{*}{ MeCP2, P250GAP,AChE } & $\uparrow$ CREB \\
\hline & & $\downarrow N R S F$ \\
\hline \multirow{2}{*}{$\operatorname{miR}-134^{36}$} & \multirow{2}{*}{ LimkI, Pum2, CREB, DCX } & $\uparrow$ mef \\
\hline & & $\downarrow Y Y \mid$ \\
\hline miR- $184^{37}$ & Akt2,Ago2 & $\begin{array}{l}\uparrow \text { STAT3 } \\
\uparrow\end{array}$ \\
\hline
\end{tabular}

Selection of the most prominent candidates, genes and miRNAs respectively, compiled from human and animal samples and postulated as potential new targets on Epilepsy therapy

$\mathrm{AChE}$, acethylcholinesterase; Ago2, argonaute; Bcl-2, b-cell lymphoma 2 gene; CDK4, cyclin-dependent kinase 4 gene; CREB, clean renewable energy bonds; DCX, doublecortin; GLUT I, glucose transporter I; LIMK I, LIM domain kinase I; MAP3k9, mitogen-activated protein kinase kinase kinase 9; MECP2, methyl CPG binding protein 2; NMDAR, n-methyl-d-aspartate receptor; mRNA, messenger RNA; P250GAP, p250GTPase-activating protein; PUM2, pumilio homolog 2; SIRTI, sirtuin I gene

On a similar way that in the research for altered genes in Epilepsy and by a similar mechanism than the described for the altered proteins on aberrant synaptic plasticity, epileptic seizures have recently 
shown to affect microRNA function. ${ }^{34-37}$ Studies in human temporal lobe epilepsy (TLE) and experimentally induced epilepsies result in changes on the level of specific miRNAs in brain specific regions or neural compartments, especially after Status Epilepticus (Table 2). Compiling different induction protocols, experimental designs and animal species used, a core set of four epilepsy related miRNAs has been suggested (miR-132, miR-134, miR-184, miR-34a) ${ }^{34-37}$ On the other hand and as the introduction of gene therapy, therapeutically miRNA targeting has, as well being proposed and its potential benefits are gaining increasing attention in multiple fields. Delivery of a miRNA inhibitor or replenishment of a lost miRNA could alter the excitability of the brain leading to less severe seizures or mitigate the downstream consequences leading to neuroprotection. ${ }^{36}$

\section{Conclusion}

The development of an epileptic syndrome includes a critical period after an initial insult and before the network reorganization and sprouting is set. The epileptogenesis time course, before the onset of spontaneous epileptiform activity, can last weeks to months (or even years) and includes a cascade of molecular and cellular events altering the excitability of the neuronal circuits involved. Thus, the experimental model development and research on this regard should be centered in this process allowing developing drugs for reversal or prevention of resistance; and development of disease-modifying therapies, inhibiting the progression of epilepsy. At the same time, efforts on the integration of translational approaches in research would facilitate disease biomarker discovery for prevention of epilepsy in patients at risk and better prognosis of treatment outcome.

\section{Acknowledgements}

None.

\section{Conflict of interest}

Author declares that there is no conflict of interest.

\section{References}

1. World Health Organization (WHO), International Bureau of Epilepsy (IBE), International League Against Epilepsy (ILAE). Atlas: Epilepsy Care in the world 2005. Geneva: World Health Organization; 2005.

2. Galanopoulou AS, Buckmaster PS, Staley KJ, et al. Identification of new epilepsy treatments: Issues in preclinical methodology. Epilepsia. 2012;53(3):571-582.

3. Bertram EH. Neuronal circuits in epilepsy: Do they matter? Exp Neurol. 2013;244:67-74.

4. Loscher W, Schmidt D. Modern antiepileptic drug development has failed to deliver: ways out of the current dilemma. Epilepsia. 2011;52(4):657-678.

5. Ben-Menachem E, Krauss GL. Responsive neurostimulation-modulating the epileptic brain. Nat Rev Neurol. 2014;10(5):247-248.

6. Duchowny M, Bhatia S. Epilepsy: Preserving memory in temporal lobectomy-are networks the key? Nat Rev Neurol. 2014;10(5):245-246.

7. Loscher W, Klitgaard H, Twyman RE, et al. New avenues for anti-epileptic drug discovery and development. Nat Rev Drug Discov. 2013;12(10):757-776.

8. Rakhade SN, Jensen FE. Epileptogenesis in the immature brain: emerging mechanisms. Nat Rev Neurol. 2009;5(7):380-391.
9. Spedding M, Neau I, Harsing L. Brain Plasticity and pathology in psychiatric disease: sites of action for potential therapy. Curr Opin Pharmacol. 2003;3(1):33-40

10. Sen A, Martinian L, Nikolic M, et al. Increased NKCC1 expression in refractory human epilepsy. Epilepsy Res. 2007;74(2-3):220-227.

11. Bender RA, Baram TZ. Hyperpolarization activated cyclic-nucleotide gated $(\mathrm{HCN})$ channels in developing neuronal networks. Prog Neurobiol. 2008;86(3):129-140.

12. Misonou H, Mohapatra DP, Park EW, et al. Regulation of ion channel localization and phosphorylation by neuronal activity. Nat Neurosci. 2004;7(7):711-718.

13. Shepherd JD, Huganir RL. The cell biology of synaptic plasticity: AMPA receptor trafficking. Annu. Annu Rev Cell Dev Biol. 2007;23:613-643.

14. Lau CG, Zukin RS. NMDA receptor trafficking in synaptic plasticity and neuropsychiatric disorders. Nat Rev Neurosci. 2007;8(6):413-426.

15. Alonso-Alonso S, Freire-Cobo C, Vszquez-Illanes MD, et al. Partial seizures induced by Latrunculin A microperfusion in the mouse hippocampus: Role of extracellular glutamate and NMDA receptors. Molecular \& Cellular Epilepsy. 2015;2: 1 .

16. Blair RE, Sombati S, Lawrence DC, et al. Epileptogenesis causes acute and chronic increases in GABAA receptor endocytosis that contributes to the induction and maintenance of seizures in the hippocampal culture model of acquired epilepsy. J Pharmacol Exp Ther. 2004;310(3):871-880.

17. Yokoi N, Fukata M, Fukata Y. Synaptic plasticity regulated by protein-protein interactions and posttranslational modifications. Int Rev Cell Mol Biol. 2012;297:1-43.

18. Kennard JT, Barmanray R, Sampurno S, et al. Stargazin and AMPA receptor membrane expression is increased in the somatosensory cortex of Genetic Absence Epilepsy Rats from Strasbourg. Neurobiol Dis. 2011;42(1):48-54.

19. Freire-Cobo C, Sierra-Paredes G, Freire M, et al. The Calcineurin inhibitor Ascomicin interferes with the early stage of the epileptogenic process induced by Latrunculin A microperfusion in rat hippocampus. $J$ Neuroimmune Pharmacol. 2014;9(5):654-667.

20. Lemtiri-Chlieh F, Zhao L, Kiraly DD, et al. Kalirin-7 is necessary for normal NMDA receptor-dependent synaptic plasticity. BMC Neurosci. 2011;12:126.

21. Kiraly DD, Lemtiri-Chlieh F, Levine ES, et al. Kalirin binds the NR2B subunit of the NMDA receptor, altering its synaptic localization and function. J Neurosci. 2011;31(35):12554-12565.

22. Melvin JJ, Huntley Hardison H. Immunomodulatory treatments in epilepsy. Semin Pediatr Neurol. 2014;21(3):232-237.

23. Legido A, Katsetos CD. Experimental studies in epilepsy: immunologic and inflammatory mechanisms. Semin Pediatr Neurol. 2014;21(3):197-206.

24. Kaminski RM, Rogawski MA, Klitgaard H. The potential of antiseizure drugs and agents that act on novel molecular targets as antiepileptogenic treatments. Neurotherapeutics. 2014;11(2):385-400.

25. Falip M, Salas-Puig X, Cara C. Causes of CNS inflammation and potential targets for anticonvulsants. CNS Drugs. 2013;27(8):611-623.

26. Ravizza T, Balosso S, Vezzani A. Inflammation and prevention of epileptogenesis. Neurosci Lett. 2011;497(3):223-230.

27. Dobolyi A, Kekesi KA, Juhasz G, et al. Receptors of peptides as therapeutic targets in epilepsy research. Curr Med Chem. 2014;21(6):764-787. 
28. Thomas RH, Berkovic SF. The hidden genetics of epilepsy- a clinical important new paradigm. Nat Rev Neurol. 2014;10(5):283-292.

29. Claes L, Ceulemans B, Audenaert D, et al. De novo SCN1A mutations are a major cause of severe myoclonic epilepsy of infancy. Hum Mutat. 2003;21(6):615-621.

30. Marini C, Darra F, Specchio N, et al. Focal seizures with affective symptoms are a major feature of PCDH19 gene-related epilepsy. Epilepsia. 2012;53(12):2111-2119.

31. Ishida S, Picard F, Rudolf G, et al. Mutations of DEPDC5 cause autosomal dominant focal epilepsies. Nat Genet. 2013;45(5):552-555.

32. Lemke JR, Lal D, Reinthaler EM, et al. Mutations in GRIN2A cause idiopathic focal epilepsy with rolandic spikes. Nat Genet. 2013;45(9):1067-1072.

33. Heron SE, Smith KR, Bahlo M, et al. Missense mutations in the sodium-gated potassium channel gene KCNT1 cause severe autosomal dominant nocturnal frontal lobe epilepsy. Nat Genet. 2012;44(11):1188-1190.

34. Nudelman AS, DiRocco DP, Lambert TJ, et al. Neuronal activity rapidly induces transcription of the CREB regulated microRNA-132, in vivo. Hippocampus. 2010;20(4):492-498.

35. Sano T, Reynolds JP, Jimenez-Mateos EM, et al. MicroRNA-34a upregulation during seizure-induced neuronal death. Cell Death Dis. 2012;3:e287.
36. Henshall DC. MicroRNAs in the pathophysiology and treatment of status epilepticus. Front Mol Neurosci. 2013;6:37.

37. Jimenez-Mateos EM, Engel T, Merino-Serrais P, et al. Silencing microRNA-134 produces neuroprotective and prolonged seizure-suppressive effects. Nat Med. 2012;18(7):1087-1094.

38. Bozzi Y, Borrelli E. The role of dopamine signaling in epileptogenesis. Front Cell Neurosci. 2013;7:157.

39. Lason W, Chlebicka M, Rejdak K. Research advances in basic mechanisms of seizures and antiepileptic drug action. Pharmacol Rep. 2013;65(4):787-801.

40. Rocha L, Alonso-Vanegas M, Villeda-Hernandez J, et al. Dopamine abnormalities in the neocortex of patients with temporal lobe epilepsy. Neurobiol Dis. 2012;45(1):499-507.

41. Ludanyi A, Eross L, Czirjak S, et al. Downregulation of the CB1 cannabinoid receptor and related molecular elements of the endocannabinoid system in epileptic human hippocampus. $J$ Neurosci. 2008;28(12):2976-2990.

42. Suls A, Mullen SA, Weber YG, et al. Early-onset absence epilepsy caused by mutations in the glucose transporter GLUT1. Ann Neurol. 2009;66(3):415-419. 